\title{
CHANGES IN ACTIVITY LIMITATIONS AND PREDICTORS OF FUNCTIONAL OUTCOME OF PATIENTS WITH SPINAL CORD INJURY FOLLOWING IN-PATIENT REHABILITATION
}

\begin{abstract}
The purpose of this study was to investigate the changes in the activity limitations of patients following in-patient rehabilitation and the factors influencing functional ability as measured by the Spinal Cord Independence Measure III (SCIM III).

A longitudinal study design was utilised to study the change in functional abilities of patients with spinal cord injury between admission and discharge. A convenient sampling strategy was employed, in which every consecutive patient admitted to the rehabilitation centre within a three month period was eligible for the study.

Demographic-, medical, and process of rehabilitation data were collected and collated from the patients' medical records using a data gathering sheet that was validated and tested for reliability.

Functional abilities were measured by the SCIM III.
\end{abstract}

Seventy-six patients met the inclusion criteria, consisting of 58 paraplegics and 18 tetraplegics. The mean age of this cohort was 34.14 years. A significant difference $(p<0.001)$ in functional ability was detected for the total sample, with only $12.5 \%$ of patients independent in walking ability and $28.12 \%$ in stair management. Four (4) factors were found to be predictors of functional outcomes on bivariate analysis, but when considered together in a multiple regression model, only functional status on admission remained correlated to functional outcomes.

Conclusion and implication for practice: Significant improvement in functional abilities of persons with spinal cord injury following in-patient rehabilitation was observed. However, mobility and stair-management limitations were the most prevalent at discharge. Lastly, a lower functional status should be better targeted to optimise functional ability in the future. Future research should be directed towards illuminating whether personal factors or rehabilitation inefficiencies are responsible for the limitations observed at discharge.

KEY WORDS: SPINAL CORD INJURY, ACTIVITY LIMITATIONS, FACTORS INFLUENCING OUTCOME.

\section{INTRODUCTION}

Spinal cord injury (SCI) is a condition that has the potential to result in severe impairments, difficulties to perform functional tasks and a compromise in quality of life (De Vivo et al, 1999). Prior to the 1940's, physicians perceived a spinal cord injury as "... an ailment not to be treated" (Hughes, 1988). Since the First World War, there has been a dramatic improvement in the management of patients with SCI in both the medical and rehabilitation spheres. However, to date, there is still no good scientific evidence for treatments conducted on patients showing an improvement in neurological impairment after acute SCI (Anderberg et al, 2007). On the contrary, there is high level evidence indicating that rehabilitation strategies could result in optimal functional outcomes (Foy et al, 2011). Despite these advancements centred towards longevity, the functioning of persons with spinal cord injury is still significantly lower than that of the rest of the population (Post et al, 1995).

Rehabilitation has become an integral part of the health care needs (Stucki et al, 2002) of persons living with spinal cord injury, with its core focus on reducing the negative consequences of the injuring event (Staines et al, 2009). From an operational perspective, the goal of rehabilitation is to establish effective and efficient practices meeting the needs of the service user and provider (Kaplan, 2007). One of the means of determining the efficacy of rehabilitation is by investigating the outcomes of

\section{Correspondence Author:}

Conran Joseph

University of the Western Cape

Physiotherapy Department

Private Bag x17

Bellville 7530

Email: conran.joseph@gmail.com 
patients, secondly by determining the factors influencing outcome and finally by aligning the process of rehabilitation (Kaplan, 2007; Hoenig et al, 1999) to address the unique human characteristics that were altered by the spinal cord injury.

With the acceptance of the International Classification of Functioning, Disability and Health (ICF) as the preferred conceptual model of describing and understanding disability, the purpose of rehabilitation has been centred towards optimising function in all dimensions of human functioning, within the categories of impairments, activity limitations and participation restrictions (Stucki et al, 2002, WHO, 2001). Within this framework, the term "activity" is defined as the difficulty an individual encounter with the execution of a task or action (WHO, 2001). A study by Dahlberg and colleagues on a cohort of patients with spinal cord injury in Helsinki found that the prevalence of activity limitations was greatest for activities related to stair management and bathing (Dahlberg et al, 2003). Literature found that prevalence data on the activity limitations of persons with SCI as essential evidence to authorities, and may assist in the planning and coordination of rehabilitation to compliment the day-to-day functional needs of the patient (Dahlberg et al, 2003).

The investigation of activity limitations could be seen as an iterative process, which could lead to the adaptation of the rehabilitation experience for specific cohorts of patients. Since an activity occurs in a context, one can infer that the execution of similar complexity of tasks within a social environment might present considerable difficulty to the patient because very few rehabilitation strategies take into account the unique context of the patient.

Kaplan considered the constituents of achieving the desired level of functioning and found that the identification of factors that may predict outcome is a critical aspect that should be determined and incorporated in appropriate health services or processes (Kaplan, 2007). Literature concerning the factors influencing outcome in patients with spinal cord injury highlights factors such as age, gender, severity and aetiology of injury to be predictive of functional recovery (Scivoletto et al, 2003). Apart from personal factors influencing outcome, studies reported the use of certain protocols, service- and process issues to account for much of the variance in functional outcome (Foy et al, 2011). Van Hedel and colleagues compared rehabilitation and the outcomes to a "black box", explaining that very little is known about the rehabilitation processes responsible for the changes in outcome (Van Hedel et al, 2012). This finding highlights the need for evaluative studies that allows us to determine factors responsible for much variance of the primary outcomes often used in rehabilitation.

Since the ICF framework provides the baseline against which pertinent outcomes should be measured, and takes into account the role of contextual factors of an individual, it is imperative to have insight into the factors predictive of functional outcome of individuals between different rehabilitation settings and patients from distinct geographical areas. In addition, the high prevalence of limitations associated with certain functional tasks will highlight the need to investigate the process and content of rehabilitation currently operational in managing those limitations. This study sets out to determine the changes in functional activity between admission and discharge, identify activity limitations at discharge and investigate the factors influencing functional outcomes of patients with a spinal cord injury following specialised inpatient rehabilitation.

\section{METHODS}

\section{Research design}

The study design was longitudinal and observational in nature, in which individuals with SCI were followed for data collection purposes for the duration of the in-patient rehabilitation, with data collected on admission and discharge. A fundamental principle of the observational approach is that it does not allow for the rehabilitation process to be influenced in any way (Smith and Smith, 2003).

\section{Research setting}

The study was conducted at a specialised rehabilitation facility in the Western Cape, South Africa. The centre admits patients with various neurological-and surgical health conditions, however this study population was limited to individuals with spinal cord injuries. Team members at this facility function within the inter-disciplinary approach. One feature of this approach is the regular (weekly) discussions regarding the patient's progress and the collective and collaborative goal- setting of individuals. In addition to the structural aspect of interdisciplinary rehabilitation at the centre, the use of the rehabilitation outcome levels (ROL's) has been regarded as standard practise and as a predictive measure of functioning along the continuum of the health condition. In short, ROL's are groupings (from level 0 to level 5) of patient problems that occur because of the consequences of the health condition along a continuum, i.e. from the acute through to the chronic phase.

\section{Population and sampling}

A sample of convenience was used to select the participants. Every consecutive patient meeting the inclusion criteria for a three month period was eligible for participation. Based on retrospective data (from the year 2010), the centre admitted more or less 320 patients with spinal cord injuries for one calendar year (personal communication, Hendry, JA). Therefore, an estimation of the desired sample size was considered for a three month period which started in November 2010 to February 2011. Eligible participants were those presenting with a spinal cord injury, aged 18 years and above, who consented in writing to participate and were proficient in English, Afrikaans or Isi-xhosa. Exclusion criteria included patients presenting with a mental disability, which was confirmed with the inability to perform a three step command test prior to the commencement of data collection. The three-month time-frame allowed for the desired sample size to be reached in order to measure the six pre-determined factors influencing functional indepen dence of patients with spinal cord injury 
at discharge. The literature recommends that for every independent variable entered at least ten respondents should be included. Thus, the three month period allowed for a minimum of 60 participants to be included (Munro, 2001).

\section{INSTRUMENTS}

\section{Data gathering sheet}

The demographic-, medical data as well as the information relating to the process of rehabilitation and rehabilitation outcome levels were collated from the medical records of the patients at discharge. The items for the demographic- and medical profile included; age, gender, marital status, diagnosis, classification of spinal cord injury. Certain items were also related to impairments following the spinal cord injury, thus muscle weakness and bladder- and bowel function were captured as either "impaired", "not impaired" or "undocumented". The documentation of the impairments was only retrieved by the doctors-, physiotherapist- or occupational therapists assessment notes on admission. Also, the variable pertaining to educational level was recorded as either primary-, secondary-or tertiary education. The items related to the process of rehabilitation and rehabilitation outcome levels were limited to the length of hospital stay (LOHS), discharge destination, health professionals involved in the management of the patients, participation in the learn-to-swim programme and outcome levels achieved on admission and at discharge.

The data gathering sheet was validated for content by two experts in the field of rehabilitation and was also found to be reliable (inter-rater) with ICC scores ranging between 0.74-1.00 for the completed sheet. Thus, the data gathering sheet presented with excellent reliability in the instance where documents were reviewed by two independent raters.

\section{Measure for functional indepen- dence and translations}

The Spinal Cord Independence Measure (SCIM) III was used to assess functional ability in this cohort of patients with spinal cord injury. This valid and reliable tool was chosen as it is the first spinal cord injury- specific measure developed for measuring functional outcomes in this particular health condition (Catz and Itzkovich, 2007). Also, all items of this measure can be mapped to the domains of the ICF, which is the conceptual model used to define activity and that was used to underpin the research findings. The SCIM III consists of 19 items, which is divided into four areas namely, self-care, respiration, sphincter management and mobility. The outcome measure is scored on an ordinal scale, with the cumulative score ranging between 0-100. Since this measure was developed with the European context in mind, it is deemed imperative to test the psychometric properties within a different context and that of the translated versions. The languages spoken by people in Cape Town are English, Afrikaans and IsiXhosa, thus the SCIM III was translated into Afrikaans and IsiXhosa. All the questionnaires were forward translated from English to Afrikaans and English to IsiXhosa respectively by one translator, thereafter the translated questionnaires were backward translation into the original language (English) by a another translator. Both translators were independent to the research project and not familiar with the medical or rehabilitation field. The SCIM III was scrutinised by the researcher and study supervisor for correctness and whether the content of each item remained the same irrespective of the translation by mapping the main construct of each item back to domains of the ICF. The reason for this process is that it is often found than when an outcome measure is translated into a different language, the translated language may express and interpret the items differently than the original version (Chang-Hoon, DongJae, Se-Kang, Dong-Jun, Hwan-Mo \& Heui-Jeon, 2006; Mkoka, Vaughan, Wylie, Yelland \& Jelsma, 2003). Therefore, based on consensus among the researcher and both translators the following changes were made to the SCIM III. The IsiXhosa translation of the SCIM III presented with the following changes. The word "oksijini" meaning oxygen from the natural environment/ atmosphere was changed to "umoya omncedisaya" which refers to artificial oxygen produced by a mechanical ventilator. This change was imperative as this would have influenced the results of respiratory function among spinal cord injuries as the aforementioned words have contrasting meanings. The word "oksijini" has an active component to breathing, whereas the desired word "umoya omncedisaya" has a passive or assisted component to respiration. The IsiXhosa word describing mobility was changed from "iintshukumo", meaning shaking, to "ukuhla usenyuka", which refers to walking as a higher functional task. Thus, the word was originally used to describe a patient's ability to walk as a means of mobilisation. The measure can be administered in different formats while maintaining good to excellent reliability, but for the purpose of this study the administration was via interview (the lead author and a trained research assistant who is proficient in English and Isi-Xhosa) which was self reported (Itzkovick et al, 2003). A recent study reported on the high correlation between the SCIM III and the SCIM self reported version (Fekete et al, 2013).

\section{Procedure and ethical statement}

All patients meeting the inclusion criteria were invited to participate in the study. After obtaining written informed consent from the patients, the medical records of the patients were perused, and a time slot was decided on for the completion of the functional outcome measure via interview. The data gathering sheet was completed on admission with the functional assessment done on admission and discharge via interview. All patients consenting to participate in the study were interviewed within two days of admission (the first two days are usually focused on the evaluation of the patient, with little to no rehabilitation input) and within the last two days prior to their discharge. All interviews lasted between 30-45 minutes. The methodology for this study had been ethically approved by the University of the Western Cape's Senate Research Committee (10/3/24), and permission was granted by the Department of Health and the Deputy Director at the Western Cape Rehabilitation Centre. 


\section{Statistical methods}

All data elements were coded and captured twice in Windows Excel 2007, and imported to SAS for analysis. The Shapiro Wilk W test was used to determine the normality of data elements. With reference to the measurement of outcome on admission and discharge, the outcome variable which was the difference in score was found to be normally distributed. Thus, the paired t-test was used to determine the significant difference in functional outcome of patients between admission and discharge, with the use of the independent $\mathrm{t}$-test for the calculated mean differences between sub-classifications of the spinal cord injured population. A 5\% significant level was used for all inferential statistics, which translates to an alpha level of 0.5 (Domholdt, 2000). Data related to the functional ability of patients with spinal cord injury were collected according to completeness of injury and each level of lesion. However, due to the small sample sizes $(<10)$ for each level, inferential statistics were not advised, due to limited power of analysis.

In response to one of the aims of the study which was to determine the factors influencing functional outcome, both linear and multiple regression models were considered to determine the relationships between independent variables and the dependent variable (functional outcome score as measured by the SCIM III). Based on the study of mean outcome scores for different categories, data were found to be abnormality distributed, thus non-parametric statistics were used. For dichotomous predictors (e.g. gender) a Wilcoxon Rank sum test was used to compare the mean outcome scores between dichotomous categories, and the Spearman's rank order correlation co-efficient was used for continuous independent variables such as length of hospital stay, age, functional outcome score on admission (0-100). A multiple regression model was fitted for four individual independent variables which were found to be associated with the functional outcome score of patients with spinal cord injury as measured by the SCIM III. The four independent variables were considered together and the final model was esta- blished through the backward elimination process.

\section{RESULTS}

\section{Recruitment of participants}

Patients with spinal cord injury accounted for $40 \%$ of the total admissions to the WCRC for the period of November 01, 2010 to January 31, 2011. Of the 93 patients with spinal cord injury, nine patients were under the age of 18 years old, two declined consent, five subjects presented with missing data and one presented with a mental illness as co-morbidities, which was considered as part of the exclusion criteria. Thus, the sample meeting the inclusion criteria was 76 patients with spinal cord injury. For the study of difference in functional outcome between admission and discharge, the sample size was reduced to 64. The reasons include the unexpected discharge of patients outside the Cape Metropole area (5 patients), suspension of patients following their weekend-pass to their residence (2 patients), death while hospitalised ( 2 patients) and the referral of patients to tertiary hospitals due to secondary complications.

\section{Profile of the participants}

The demographic data are presented in Table 1. The mean age of the patients was 34.14 years (SD: 12.42), with more paraplegic patients $(76.3 \%)$ following injury to the spinal cord. The age of patients ranged from as young as 18 to 81 years of age. Most of the patients were single at the time of injury.

\section{Process of rehabilitation}

All patients with spinal cord injury were managed by the nurse and physiotherapist, with most of them also managed by the doctor, occupational therapist and social worker. The length of hospital stay in days was longer for the cohort with tetraplegia (85.6), with the majority of patients discharged to their long-term residence. Furthermore, less than 20\% of the sample was referred for followup medical intervention at either the institution's out-patient department or another health care facility. Several of the patients $(40.8 \%)$ were admitted with a health status corresponding to outcome level two, and $69.7 \%$ of patients were discharge with an outcome level greater than three (Table 2).

\section{Functional outcomes of patients according to the Spinal Cord Inde- pendence Measure III}

Table 3 illustrates the statistical difference in functional outcome between admission and discharge, and provides the level of significance for the total sample as well as the sub-classifications of SCI. The sum score on the SCIM III at discharge, for both the total sample and sub-classifications, was significantly larger than that on admission as determined by the paired t-test. The significant difference between the means scores of those with paraplegia and tetraplegia indicated that the mean score of the paraplegic population, on both admission and discharge, was significantly higher than the tetraplegic group with $p=0.023$ and $p=0.007$ respectively.

Table 1: Demographic data of the participants with spinal cord injury $(n=76)$

\begin{tabular}{|l|l|}
\hline $\begin{array}{l}\text { Demographic and admission data } \\
\text { - Spinal cord injury }\end{array}$ & $34.14(12.4) ; \min =18, \max =81$ \\
\hline $\begin{array}{l}\text { Gender: Male to female ( } ; \%) \\
\text { - Spinal cord injury }\end{array}$ & $56(73.7) ; 20(26.3)$ \\
\hline $\begin{array}{l}\text { Marital status: married ( } \mathrm{n} ; \%) \\
\text { - Spinal cord injury }\end{array}$ & $14(18.4)$ \\
\hline $\begin{array}{l}\text { Classification of Spinal cord injury }(\mathrm{n} ; \%) \\
\text { - Paraplegia } \\
\text { - Tetraplegia }\end{array}$ & $58(76.3)$ \\
\hline
\end{tabular}


Table 2: The process of rehabilitation of pateints with spinal cord injury

\begin{tabular}{|l|l|}
\hline Process of rehabilitation & \\
\hline Health professionals seen by patients (n; \%) & $6(9.4)$ \\
- Psychologist & $63(98.4)$ \\
- Doctor & $64(100.0)$ \\
- Nurse & $62(96.9)$ \\
- Occupational therapist & $64(100.0)$ \\
- Physiotherapist & $4(6.3)$ \\
- Speech therapist & $60(93.8)$ \\
- Social worker & $10(15.6)$ \\
- Dietician & \\
\hline Length of hospital stay (days; SD) & $73.11(43.7)$ \\
- SCl population & $68.32(40.1)$ \\
- Paraplegia & $85.65(50.4)$ \\
- Tetraplegia & \\
\hline Discharge destination with referral (n; \%) & $61(80.3)$ \\
- Home & $4(5.3)$ \\
- Health care facility & $9(11.8)$ \\
- WCRC OPD & $2(2.61)$ \\
\hline Rehabilitation outcome levels (\% admission; discharge) & $1.32(0.0)$ \\
- Level 0 & $30.26(0.0)$ \\
\hline - Level 1 & $40.79(1.3)$ \\
\hline
\end{tabular}

Table 3: SCIM III mean scores, standard deviation and mean difference of the total sample- and sub-classification of $\mathrm{SCl}$

\begin{tabular}{|l|l|l|l|}
\hline & $\begin{array}{l}\text { Admission } \\
\text { Mean (SD) }\end{array}$ & $\begin{array}{l}\text { Discharge } \\
\text { Mean (SD) }\end{array}$ & Significance \\
\hline Variable & $48.0(24.3)$ & $67.1(23.0)$ & $\mathrm{p}<0.001$ \\
\hline $\begin{array}{l}\text { SCIM score III of } \\
\text { total sample }\end{array}$ & $53.7(22.2)$ & $73.2(18.2)$ & $\mathrm{p}=0.006$ \\
\hline $\begin{array}{l}\text { SCIM III score of } \\
\text { paraplegics }\end{array}$ & $41.6(30.6)$ & $53.0(33.3)$ & $\mathrm{p}=0.033$ \\
\hline $\begin{array}{l}\text { SCIM III score of } \\
\text { tetraplegics }\end{array}$ & & & \\
\hline
\end{tabular}

1. The sample $(n)$ of those with paraplegia was $n=58$ and $=52$ on admission and discharge respectively.

2. The sample $(n)$ of those with tetraplegia was $n=18$ and $=15$ on admission and discharge respectively.
Independent execution of tasks as derived from the SCIM III on admission and at discharge

Figure 1 shows the degree of independent execution of tasks on admission was greatest for respiration, feeding and grooming with $98.7 \%, 79.0 \%$ and $76.3 \%$ of patients fully functional with the respectively tasks. The tasks in which most patients were still dependent on and needed assistance with, were related to mobility (indoors, along moderate distances and mobility outdoors), stair management and transfer from floor to wheelchair. The greatest increases in the independent execution of tasks between admission and discharge were found for bathing of the lower limbs, transfer from the wheelchair to the car, and toileting with $43.7 \%, 43.3 \%$ and $43.0 \%$ of additional patients who were not independent with the execution of the respective tasks on admission.

Factors predictive of outcome for patients with spinal cord injuries

The SCIM III was used to measure functional abilities in the cohort of patients with spinal cord injuries. This measure was considered as an ordinal scale with the score ranging between 0-100 in increments of 5 points. The list of independent variables (which was both continuous and categorical) that was considered for the bivariate analysis is summarised below in table 4.

The results indicate that higher activity scores on admission, younger age, and shorter length of hospital stay were found to be correlated with better functional abilities, as measured by the SCIM III, at discharge. The inverse relationship between age and length of hospital stay and the functional outcome score at discharge is evident in the Spearman's correlations of -0.30 and -0.28 respectively. The only categorical variable that was found to be correlated with functional ability were the absence of participation in the learn-to swim programme offered at the centre, as this was confirmed by a $p=0.013$. The relationship was strong between the activity levels on admission as measured by the SCIM III compared to the activity level at discharge. This relationship was confirmed by Spearman's $r=0.66$ and a $\mathrm{p}<0.001$. 




* 1- Feeding; 2-Bathing upper body; 3-Bathing lower body; 4- Dressing upper body; 5- Dressing lower body; 6- Grooming; 7- Respiration; 8- Bladder management; 9- Bowel management; 10- Use of toilet; 11- Mobility in bed and action to prevent pressure sores; 12 - Transfer from bed to wheelchair; 13- Transfer from wheelchair-toilet and tub; 14- Mobility indoors; 15- Mobility for moderate distances; 16- Mobility outdoors; 17- Stair management; 18- Transfer from wheelchair to car; 19- Transfer from ground to wheelchair

Figure 1: Independent execution of tasks as derived from the SCIM III on admission and at discharge

Table 4: Bivariate testing of variables that could influence activity as measured by the SCIM III at discharge ( $\mathrm{n=64)}$

\begin{tabular}{|l|l|l|l|l|l|}
\hline Variable & $\begin{array}{l}\text { Nature of } \\
\text { variable }\end{array}$ & Measurement & Test & $\begin{array}{l}\text { Spearman's } \\
\text { correlation } \\
\text { value }\end{array}$ & Significance \\
\hline Gender & Categorical & Male; Female & Wilcoxon Rank & N/A & $p=0.97$ \\
\hline Age & Continuous & Years & Spearman's rho & $r=(-) 0.30$ & $p=0.018$ \\
\hline LOHS & Continuous & Days & Spearman's rho & $r=(-) 0.28$ & $p=0.034$ \\
\hline SCIMIII & Continuous & $0-100$ & Spearman's rho & $r=0.66$ & $\mathrm{P}<0.001$ \\
\hline Learn to swim & Categorical & Yes; No & Wilcoxon Rank & N/A & $p=0.013$ \\
\hline Muscle weakness & Categorical & Yes; No & Wilcoxon Rank & N/A & $p=0.62$ \\
\hline Education & Categorical & Low; High & Wilcoxon Rank & N/A & $p=0.10$ \\
\hline
\end{tabular}

* Key: LOHS-length of hospital stay; SCIM III-Spinal Cord Independence Measure version III on admission; N/A- Not applicable

Table 5: Summary of remaining variable in the Multiple predictor model

\begin{tabular}{|l|l|l|l|l|}
\hline Variable & $\begin{array}{l}\text { Parameter } \\
\text { Estimate }\end{array}$ & $\begin{array}{l}\text { Standard } \\
\text { Error }\end{array}$ & F Value & Pr $>$ F \\
\hline Intercept & 51.158391 & 8.17371 & 39.17 & $<.0001$ \\
\hline $\begin{array}{l}\text { Outcome score } \\
\text { admission }\end{array}$ & 0.57208 & 0.09795 & 34.11 & $<.0001$ \\
\hline
\end{tabular}




\section{Multiple predictor model consi- dered for spinal cord injury}

Since there were two variables that showed a relatively strong relationship to outcome level scores (as measured by the SCIM III) and two other variables showing a slightly weaker relationship, but yet significant, these variables were considered together in a multiple regression model. (See Table 5).

During the backward elimination process age, participation in the learnto-swim programme and length of hospital stay were insignificant predictors when considered with only the outcome score on admission. With regards to the parameter estimate of the outcome score on admission, the analysis indicates that for every one point (as achieved on the SCIM III) on admission, the predicted value of the outcome score at discharge (as measured by the SCIM III) will have an additional 0.57 points.

\section{DISCUSSION}

The aim of this study was twofold; firstly to determine the activity limitations and secondly to identify the predictors of functional outcome as measured by the SCIM III. The mean intake SCIM III score for this cohort of patients was 48.03, which clearly shows that participants in the present study had a much higher level of functioning than patients with SCI in Europe, with a mean intake SCIM II score of 32.00 (Wirth et al, 2008). The latter reported study demonstrated change in functional status at three- and six months with median SCIM II scores of 60 and 71 respectively. It is important to note that the mean LOHS of patients with spinal cord injury in the current study was 73.11, which loosely converts to more or less two and a half months of hospitalisation, but with a mean SCIM III score of 67.07 (SD: 23.0), which was higher than the score obtained after three months in the study conducted by Wirth et al (Wirth et al, 2008). A possible explanation for this difference in functional status between the current study and the study by Wirth and colleagues could be that the sample in the latter study comprised of more complete tetraplegics ( $42 \%$ of sample), whereas the current study included more paraplegics $(76.3 \%)$, or the criteria for admission to the centre could have accounted for this difference in which only persons with no complications or secondary complications are admitted and those who could benefit from inpatient rehabilitation.

The highest levels of independence were for the items respiration, feeding and grooming. These findings are consistent with the results from a study conducted in eight rehabilitation centres in the Netherlands (Post et al, 2005), except for the item relating to respiration. The reason for this being that the latter study utilised the FIM, which omits items related to respiratory function. Looking at individual items, the majority of the patients with spinal cord injury in the current study experienced high levels of limitation with mobility (walking) and stair management at discharge, nevertheless the difference was statistically significant between the points of data collection. Similar findings were found elsewhere (Wirth et al, 2008; Chan and Chan, 2005). Optimal outcome levels have not yet been achieved at the time of discharge from rehabilitation for this cohort of patients. The tacit knowledge as derived from the items of the standardised outcome measure and the global rating score of the rehabilitation outcome levels suggests that there are limitations that could be addressed. However, the authors are in agreement that a qualitative inquire would yield considerably more trustworthy data of the nature of the participants' problems and the barriers to their achievement of certain activities or engagement in certain life situations.

The high level of disability associated with mobility (specifically relating to walking ability) is slightly ambiguous as the nature and extent of the spinal cord injury were not taken into account, irrespective of the independent execution of mobility tasks using a wheelchair. Despite the recognition of the SCIM III as the gold standard measure in the evaluation of the functional ability of persons with SCI, the measure lacks responsiveness. For example, if a patient is capable of being mobile in a wheelchair, without physical assistance, the outcome measure will continue to indicate an inferior score compared to someone walking with assistance and personal support. Despite this limitation, the need for follow up rehabilitation post discharge is implied since studies found significant improvement in mobility, particularly within the mobility construct, for up to six months after injury (Wirth et al, 2008). Since patient with spinal cord injuries are inherently limited in mobility tasks, the author and other published authors (Ullrich et al, 2012) are concerned about current measuring tools, such as the Functional Independence Measure and SCIM III that do not consider the level of lesion as a legitimate level of functioning with predefined capabilities for that specific individual. Therefore, floorand ceiling effects of outcome measures are almost always present. The recommendation of a recent study by Ullricht and colleagues advocate for the inclusion of patients with spinal cord injuries in the decision-making process of which constructs of functioning should be included for the evaluation of the holistic impact of such an injury on the individual (Ullrich et al 2012).

With the focus on predictors of functional outcome in this cohort of patients, the current study found age to be inversely related to functional outcome. Case controlled studies conducted by Scivoletto et al (2003) and Seel et al (2001) found that independence of daily living measures were significantly greater $(p<0.001)$ for the younger age group and the young to middle age group respectively. The achievement of better functional outcomes in the younger age group could be due to the traumatic nature of the lesions, which have been associated with a higher probability of sustaining complete motor lesions of the thoracic and lumbar levels, whereas non-traumatic lesions are more common in older patients and have higher frequencies of incomplete tetraplegia (Scivoletto et al, 2003). Apart from age, the current study and numerous published reports found length of hospital stay to be a predictor of functional outcome at discharge (Sipski et al, 2004; Scivoletto et al, 2003). The current study found a significant correlation between LOHS and functional outcome at discharge (spearman's rho-0.28; $\mathrm{p}=0.035$ ), which in this instance could be the result 
of the sample consisting of more paraplegics $(76.31 \%)$ than tetraplegics (Post et al, 2005). Although the mean length of hospital stay in the current study between those with paraplegia (68.53; SD 50.5) and tetraplegia (85.62; SD 40.6) was statistically insignificant, the difference of 17 days has clinical meaning and relevance as cost of healthcare is usually estimated based on the length of hospital stay in days (Winslow et al, 2002). The inability to detect the significant difference in LOHS between those with paraplegia and tetraplegia could be due to the large standard deviation, which could be influenced by the small size that reduces the power of analysis. The shorter length of hospital stay as an indicator for better functional outcomes should be analysed with caution, as it could simply imply that those with paraplegia reach their level-specific outcomes sooner than those with tetraplegia, due to the initial level and severity of the injury and age.

Literature highlights that by combining land-based rehabilitation with aquatic physical therapy, better outcomes may be achieved in recovery of function in adults with acquired neurological impairments (Degano and Geigle, 2009). The current study findings provided evidence of the effect of a learn-to-swim programme /aquatic therapy on the functional outcomes of those patients with spinal cord injury. The bivariate analysis demonstrated that those who attended the learn-to-swim programme had a significantly higher functional outcome $(p>0.013)$ at discharge than those who did not attend, whereas an insignificant difference between the mean functional outcome scores of the two independent samples (those who did attend versus those who did not attend) was found on admission.

Results from the multiple regression model, which used a backwards elimination procedure indicated that only functional outcome to be significant in the model. Similar results are found elsewhere (Burnett et al, 2000). Based on theoretical principles, discharge planning should be guided by functional outcome and not health care cost (Visagie, 2012). On the basis of this investigation, with the author aware of the limitations of this model (small sample size which led to the inclusion of only certain variables), the functional outcomes at discharge could be predicted. This model provides relevant health care professionals and patients to aid in the identification of factors which could impede functional outcomes and the opportunity to aggressively manage and appropriately refer patients to other relevant professionals. Since the functional outcome on admission accounted for almost half $46 \%$ of the variance in the overall functional score at discharge, it is recommended that rehabilitation professionals administer the SCIM III on admission, which is an easy tool to use, in order to set realistic goals and to decide on the appropriate rehabilitation services and processes for the patient.

In conclusion, this study clearly highlights the changes in functional abilities and activity limitations in patients with spinal cord injury, following specialised in-patient rehabilitation. Based on the outcomes, limitations in mobility and stair management are still most prevalent at discharge. Since activity limitations influence participation, given that participation restrictions are difficult to be evaluated and addressed outside of the patient's social milieu, it is essential that rehabilitation services should be available, not necessarily on an in-patient basis, following discharge from the specialised rehabilitation unit. Furthermore, the predictors of functional outcome of this particular study should serve as a basis for targeting and appropriately manage the factors that could negatively impact the functional outcome of patients with spinal cord injury.

\section{LIMITATIONS OF THE STUDY}

This study did not determine the completeness of the lesion and failed to capture the neurological classification according to ASIA. Consequently, comparison of data will be negatively influenced. Furthermore, the exclusion of patients under the age of 18 and those with co-morbidities could have influenced the normality of the data.

\section{ACKNOWLEDGEMENT}

The authors would like to thank South Africa Netherlands research Programme on Alternatives in Develop- ment (SANPAD) for financial support and for the patients for their participation in this study.

\section{REFERENCES}

Anderberg L, Aldskogius H, Holtz A 2007 Spinal cord injury--scientific challenges for the unknown future. Ups J Med Sci 112: 259-288

Burnett DM, Kolakowsky-Hayner SA, Gourley EV, Cifu DX 2000 Spinal cord injury "Outliers": An analysis of etiology, outcomes, and length of stay. J neurotrauma 17(9): 765-772

Catz A, Itzkovich M 2007 Spinal Cord Independence Measure: Comprehensive ability rating scale for the spinal cord lesion patient. J Rehabil Res Dev 44(1): 65-68

Chan SC, Chan AP 2005 Rehabilitation outcomes following traumatic spinal cord injury in a tertiary spinal cord injury centre: a comparison with an international standard. Spinal Cord; 43(8): 489-498

Dahlberg A, Kotila M, Kautiaien H, Alaranta H 2003 Functional independence in persons with spinal cord injury in Helsinki. J Rehabil Med 35: 217-220

Degano AC, Geigle PR 2009 Use of aquatic physical therapy in the treatment of balance and gait impairments following traumatic brain injury: A case report. J Aquatic Phys Ther 17: 16-21

DeVivo MJ, Krause JS, Lammertse DP 1999 Recent trends in mortality and causes of death among persons with spinal cord injury. Arch Phys Med Rehabil 80: 1411-1419

Domholdt E 2000 Physical Therapy Research: Principles and Application ( $2^{\text {nd }}$ ed.) WB Saunders Publishers: Philadelphia

Fekete C, Eriks-Hoogland I, Baumberger M, Catz A, Itzkovich M, Luthi H, Post MWM, von Elm E, Wyss A, Brinkhof MWG 2013 Development and validation of a self-report version of the Spinal Cord Independence Measure (SCIM III). Spinal Cord 51: 40-47

Foy T, Perritt G, Thimmaiah D, Heisler L, Offutt J, Cantoni K, Hseih C-H, Gassaway J, Ozelie R, Backus D 2011 Occupational therapy treatment time during in-patient spinal cord injury rehabilitation. J Spinal Cord Med 34(2): 162-175

Hoenig H, Horner RD, Duncan PW, Clipp E, Hamilton B 1999 New horizons in stroke rehabilitation research. J Rehabil Res Dev 36(1): 19-31 
Hughes JT 1988 The Edwin Smith Papyrus; an analysis of the first case reports of spinal cord injuries. Paraplegia 26:71-82. p.72

Itzkovich M, Tamir A, Philo O, Steinberg F, Ronen J, Spasser R 2003 Reliability of the CatzItzkovich spinal cord independence measure assessment by interview and comparison with observation. Am J Phys Med Rehabil 82: 267-272

Kaplan SL 2007 Outcome measurement and Management: First Steps for the Practicing Clinician. ppl 55-63. F.A. Davis Company, United States of America

Munro BH 2001 Statistical methods for health care research. Lippincott, New York, Baltimore

Post M, Dallmeijer A, Angenot E, van Asbeck F, van der Woude L 2005 Duration and functional outcome of spinal cord injury rehabilitation in the Netherlands. J Rehabil Res Dev 42(3): 75-86

Post MWM, van Asbeck FWA, van Dijk AJ, Schrijvers AJP 1995 Dutch interview-version of the Barthel Index validated for persons with Spinal Cord Injury (In Dutch). Nederlands Tijdshrift voor Geneeskunde 139: 1376-1380
Scivoletto G, Morganti B, Ditunno P, Ditunno JF, Molinari M 2003 Effects on age on spinal cord lesion patients' rehabilitation. Spinal Cord 41(8): 457-464

Seel RT, Huang ME, Cifu DX, KolakowskyHayner S A, McKinley WO 2001 Age-related differences in length of stays, hospitalization costs, and outcomes for an injury-matched sample of adults with paraplegia. J Spinal Cord Med 24(4): 241-250

Sipski ML, Jackson AB, Gómez-Marín O, Estores I, Stein A 2004 Effects of gender on neurologic and functional recovery after spinal cord injury. Arch Phys Med Rehabil 85(11): 1826-1836

Smith F, Smith J 2003 Clinical Research (1 ed.). ppl 34- 54. BIOS Scientific Publishers. Oxford

Staines WA, McIlroy WE, Brooks D 2009 Functional impairments following stroke: Implications for rehabilitation. Current Issues in Cardiac Rehabilitation and Prevention 17(1): 5-8

Stucki G, Ewert T, Cieza A 2002 Value and application of the ICF in rehabilitation medicine. Disabil Rehabil 24(17): 932-938
Ullrich PM, Spungen AM, Atkinson D, Bombardier $\mathrm{CH}$, Chen Y, Erosa NA, Groer S, Ottomanelli L, Tulsky DS 2012 Activity and participation after spinal cord injury: Stateof-the-art report. J Rehabil Res Dev 49(1):155-74

Visagie, S. (2012). Rehabilitation outcomes of persons with complete paraplegia at a private rehabilitation hospital. South African J Physiother 68(1): 1-7

Winslow C, Bode RK, Felton D, Chen D, Meyer PR 2002 Impact of respiratory complications on length of stay and hospital cost in acute cervical spine injury. Chest 121: 1548-1554

Wirth B, van Hedel H, Kometer B, Dietz V, Curt A 2008 Changes in activity after a complete spinal cord injury as measured by the spinal cord independence measure II (SCIM). Neurorehabil Neural Rep 22: 279-287

World Health Organisation. International Classification of Functioning, Disability and Health (ICF) 2001. Short version. Geneva: WHO 